\title{
Increment detection thresholds during binocular rivalry suppression ${ }^{1}$
}

\author{
RICHARD WALES AND ROBERT FOX ${ }^{2}$ \\ VANDERBILT UNIVERSITY
}

In two experiments, two-choice forced-choice duration thresholds for increment test flashes were estimated during phases of rivalry suppression and nonsuppression and for a nonrivalry monocular control condition. In both experiments thresholds of both eyes of each $S$ were measured and, to maximize correct detections, feedback was given after every trial and $S$ s were relieved of the task of continually reporting changes in rivalry phases. Results of both experiments support the conclusion that suppression constitutes an elevation in threshold, on the order of $.5 \mathrm{log}$ units relative to thresholds found during nonsuppression and monocular conditions. These data, in concert with others, reinforce the general conclusion that rivalry suppression is an inhibitory state that nonselectively attenuates all classes of inputs falling within the spatial boundaries of the suppressed target.

The phenomenal suppression characteristic of binocular rivalry is an impressive phenomenon that reveals the presence of an inhibitory component within the binocular visual system. Yet relatively little is known about the mechanism responsible for such suppression.

To investigate the operation of the suppression mechanism, several recent investigations have measured the sensitivity of the eye during suppression by a test-probe method and found that phenomenal suppression is accompanied by a reduction in sensitivity to the test probe. Using detection of a target put into motion during suppression as the test, Fox and Check (1968) found that detection of movement of a contour is attenuated during suppression. Further, forced-choice letter recognition thresholds are elevated during suppression even though the configurations of the letters are quite dissimilar to the rivalry targets (Fox \& Check, 1966).

While these results indicate that the suppression state is inhibitory, many questions about the functional characteristics of suppression remain unanswered. One important question concerns the extent to which suppression is selective. Two alternative hypotheses can be entertained. The first hypothesis contends that suppression selectively attenuates only certain classes of inputs while exerting no effect on other classes of inputs. The second hypothesis holds that the suppression process is nonselective, i.e., suppression attenuates all inputs regardless of their physical properties.

Alth, ough the fact that moving targets and ietter forms are attenuated by supprcssion is consistent with the nonselective hypothesis, both types of targets are composed of distinct contours, and it is possible that suppression acts only upon contoured targets. If that is so, then the detectability of essentially contour-free targets such as a light flash would not be altered by the suppression state, a result that would require rejection of the nonselective suppression hypothesis.

Since a contour can be defined as an abrupt spatial change in brightness, a flash of light meets that definition; therefore, it may be misleading to suggest that light flashes are contour-free. A more nearly correct statement is that detection of a threshold light flash involves only a minimal amount of processing of information about contour since the very brief change in brightness does not allow for the formation of an edge or a specific shape, nor are the variables of edge and shape essential for satisfactory detection performance. But for the correct recognition of a form, considerably more contour information must be processed since the resolution of edge and shape is essential for satisfactory performance. The distinction being made here is not really between the presence or absence of contour, but rather between the amount of contour information that must be extracted. That difference seems sufficient to support the conjecture that form recognition involves more stages of the perceptual system than does detection. If so, it seems reasonable to suspect that suppression may interfere with the recognition stages, but not the detection stages.

Accordingly, the present experiments were designed to examine this possibility. The basic question concerned the degree to which the detectability of a test probe, in this case a light flash imposed on a rivalry target, is attenuated during the suppression state of rivalry when the conditions for a correct detection are optimum. The experimental design maximized the possibility of a correct detection in the following ways: (1) A forced-choice response in conjunction with correct feedback following each trial was utilized. Both theoretical and empirical considerations suggest that the coupling of the forced-choice response with correct feedback maximizes the probability of a correct detection (e.g., Blackwell, 1953; Cornsweet \& Pinsker, 1965). (2) The S was not required to report continuously the rivalry alternations. Instead, he initiated each trial whenever clear suppression and nonsuppression states were present. This arrangement, which closely approximates conventional psychophysical detection methods, allowed the $S$ to direct full attention to the detection task.

\section{EXPERIMENT 1}

\section{Method}

Subjects. Two young males, DC and JR, 20 and 23 years of age, respectively, served as paid volunteers. Both Ss had normal uncorrected vision, as measured by the Modified Orthorater tests, and both were experienced in observing rivalry but were naive regarding the purpose of the experiment.

Apparatus. The basic optical system was a Wheatstone stereoscope constructed from optical bench components. The left-eye and the right-eye stimulus fields that contained the rivalry-inducing targets were 6-deg squares located $75 \mathrm{~cm}$ from each eye. Fields were transilluminated by incandescent bulbs; control of luminance was achieved by neutral density filters. The entire apparatus, including light baffles and septum, was enclosed in a light-tight housing. At the viewing port of the housing, a headrest and chin cup were located and a trial frame with many adjustable features was permanently fixed into position. The trial frame contained 2-mm artificial pupils formed with an extruded conical section that located the physical aperture as close as possible to the cornea. The stimulus holders, mirrors, and other optical components of the stereoscopy were adjustable so that fusion could easily be obtained for each $S$ without strain. These components also permitted the maintenance of constant illumination of all displays.

The configuration of the rivalry targets is illustrated in Fig. 1. The right-eye target consisted of a foveally located 1-deg solid black disk surrounded by a concentric 


\section{STIMULUS CONFIGURATIONS, RIGHT AND LEFT EYE}
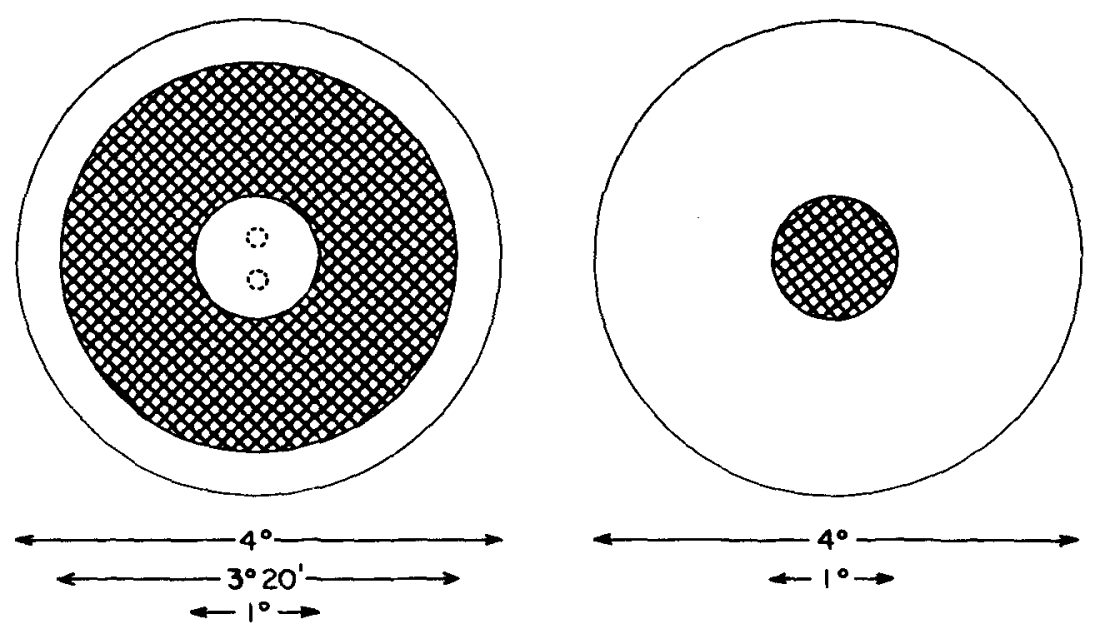

SYMBOL : SHOWS POSITIONS IN WHICH TEST FLASH COULD OCCUR

4-deg white disk, with the remainder of the field remaining black. The left-eye target consisted of a foveally located 1-deg solid white disk surrounded by a concentric 3-deg 20-min solid black disk surrounded in turn by a concentric 4-deg solid white ring, with the remainder of the field being solid black. When the targets were fused, the central 1-deg disks were coincident, as were the outer edge of the white ring of the right-eye target and the outer edge of the 4-deg white disk of the left-eye target. These outer rings served to maintain fusion of the targets, while the central 1-deg disks engaged in active rivalry; since the $S$ s reported only on the rivalry of the central disks, they were called the rivalry targets. The targets were made from high-contrast negatives (Kodalith), the contrast of which was further increased by painting the black: area of the negatives with opaque paint. The negatives were mounted on translucent: Plexiglas sheets, a combination that yielded homogeneous white areas within the clear portions of the negative and essentially opaque areas for the black portion of the negatives. The luminance of the white areas was $3 \mathrm{fL}$ for both eyes and both Ss; the luminance of the black areas was less than $.001 \mathrm{fL}$. All luminance values were measured and checked periodically with a Pritchard Spectra Spot Photometer, Model 1970-PR.

The test-probe stimuli used for measuring the detection thresholds were $8.5-\mathrm{min}$ circular flashes of light superimposed optically on the plane of the central 1-deg rivalry targets. The basic psychophysical procedure employed a spatial two-choice forced-choice task in which the flashes could appear either within the upper half or within the lower half of the central rivalry target of each eye. The separation of the flashes measured from their borders nearest one another was $12 \mathrm{~min} 18 \mathrm{sec}$.

The flashes were produced by imposing a high-voltage square-wave pulse across standard fluorescent lamps, following a circuit described by Gerbrands and Stevens (1964). The lamps were Sylvania F15T8-CW cylindrical tubes, one lamp being used for each eye separately. To keep the lamps partially ionized, a $10-\mathrm{V}$ dc current was always present across the filament of each lamp. A very well regulated dc power supply provided the 400-V striking voltage for the lamps and an electronic stimulator (AEL Model 104A) controlled the high voltage gate circuit and the time duration of the striking voltage. The lamps were selected from a batch of lamps on the basis of their reliability of firing and their sharp rise and decay times. For both lamps, the rise to maximum amplitude was of the order of $1.5 \mathrm{msec}$; decay of maximum amplitude to $90 \%$ occurred within $1 \mathrm{msec}$. The wave form for the flashes of both lamps was continuously monitored by an oscilloscope. Phototubes (P929) were used to adjust and monitor the relative light energies of the lamps. Each lamp for each eye was mounted in a separate light house. In the light house, a lens collimated and magnified the light beam, the magnification serving to eliminate luminosity gradients in the beam. Directly in front of this lens a set of neutral density filters were fixed in position. These filters served to equate
Fig. 1. The configuration of the rivalry targets used in both experiments. Cross hatching indicates black areas; absence of hatching indicates white areas.

roughly the luminance of the two lamps. Outside the light house and in the optic path of the light beam an array of neutral density filters was located on rotary wheels so that fine adjustments of luminance could be made throughout the experiment proper. To eliminate electronic interaction between the lamps that might have varied time and amplitude characteristics, both lamps always fired simultaneously by means of the same gating system, so that onset and duration were identical. To allow for stimulation of a single eye and to vary the spatial position of the test flash, occluders and test flash apertures were located in front of the rotary filter wheels. The occluders operated to reciprocally block the optic path of one lamp or the other. The circular apertures that admitted the test flash could be moved up or down to vary the spatial location of the test flash so that it would appear either in the upper half or the lower half of the rivalry target.

To mask the sounds of the movements of the occluder, the aperture, and the rotary filter wheels, a moderate level of white noise was delivered to $S$ through stereo headphones. To provide feedback after every trial a tone burst was superimposed upon the white noise. A distinctive high tone indicated a correct judgment and a distinctive low tone indicated an incorrect judgment.

Design and procedure. Prior to obtaining thresholds under rivalry conditions, monocular thresholds for the light flash were obtained for each eye of each $S$. The test flashes were imposed upon the rivalry target appropriate for the eye under test, i.e., left-ye target or right-eye target, while the other eye remained open and viewed a contourless, white, homogeneously lit 6-deg square field with luminance equal to that of the white portions of the corresponding rivalry target.

The stimulus conditions for the monocular control thresholds were selected to simulate as far as possible the conditions of threshold measurement during rivalry. To that end, the luminance level presented to the eye not being tested was the same as the luminance used in the rivalry condition. Further, to approximate the state of uncertainty present during rivalry about which eye was to receive the test flash, Ss were told to keep both eyes open because the test flash might occur in either eye, and to maintain that set flashes were occasionally presented to the eye not under test. It should be noted that the 
Table 1

Experiment 1: Proportion of Correct Detections Under Suppression (SP) and Nonsuppression (NS) Conditions and Differences Obtained

\begin{tabular}{|c|c|c|c|c|c|c|}
\hline \multirow[b]{2}{*}{$\mathbf{S}$} & \multicolumn{3}{|c|}{ Left Eye } & \multicolumn{3}{|c|}{ Right Eye } \\
\hline & SP & NS & Diff. & SP & NS & Diff. \\
\hline DC & .64 & .90 & $.26 * *$ & .63 & .72 & $.09 *$ \\
\hline JR & .51 & .90 & $.39 * *$ & .59 & .71 & $.12 * *$ \\
\hline
\end{tabular}

$* p<.05 ; * *<<.01$

binocular combination of the rivalry target in the eye under test with the homogeneous field in the eye not under test did not produce patent rivalry. The rivalry target always dominated the homogeneous field.

Although the monocular conditions we employed seem appropriate to the purpose of the experiments, the general question of what constitutes a proper monocular condition is difficult to answer, for, as Verhoeff (1935) and Woodworth (1938) point out, closing one eye does not preclude the interaction of the resulting dark field with the bright field of the open eye. In fact, after prolonged observation, occasional suppression of the view of the open eye by the closed eye can be seen. It would seem that any difference in stimulation between the eyes has the potential for inducing rivalry; the probability of patent rivalry would be a function of the variables of the specific experimental situation. Even when there are no differences in stimulation between the eyes, for example, suppose both eyes fuse identical contours, it is possible that the eyes are engaged in inhibitory interactions-a hypothesis explicitly incorporated within suppression theories of binocular combination (e.g., Verhoeff, 1935). These considerations suggest that selection of monocular viewing conditions for comparison with binocular conditions should be made within the context of a general theory of binocular vision, and that the inability to specify unambiguously the appropriate condition reveals the incomplete status of the theory.

The psychophysical method employed to estimate thresholds was a random double staircase method whereby $S$ had to make a forced-choice judgment on every trial as to the location of the test flash within the rivalry target; the a priori probability of the test flash being in the upper half or the lower half of the rivalry target was .50. After each choice, S received correct information about the location of the flash for that trial. The rule structure of the staircase method was set so that the terminal value of the test flash energy yielded correct detection $75 \%$ of the time.

To vary test-flash energy for the right-eye thresholds, the duration of the flash was varied in 5-msec steps with the luminance of the flash held constant. To vary the energy level of the test flash for the left eye, the duration of the flash for the left eye was held constant at the value found for the right-eye threshold and the luminance of the left-eye flash was varied in .1-log-unit steps. Luminance was varied for the left eye, rather than duration, because this was more convenient. The threshold values for the two Ss were as follows: For JR, the test flash duration was $70 \mathrm{msec}$; the luminance for the right eye was $.9 \mathrm{fL}$ and for the left eye, $2.9 \mathrm{fL}$. For DC, the test flash duration was $65 \mathrm{msec}$; the luminance value for the right eye was $.8 \mathrm{fL}$ and for the left eye, $4.4 \mathrm{fL}$.

These values were used during data collection under the rivalry conditions. During the rivalry conditions, the Ss did not actually report continuously on the rivalry fluctuations. Instead, they pressed a switch whenever they had clear nonsuppression of either the left-eye target or the right-eye target. Pressing the switch activated automatic circuitry that delivered the test stimulus $250 \mathrm{msec}$ later. The determination of the state in which $S$ pressed the switch, either the left-eye nonsuppression state or the right-eye nonsuppression state, was governed by instructions from $E$, who had a predetermined random schedule of left-eye and right-eye presentations. After each test-flash presentation, which constituted a trial, $S$ indicated whether or not the rivalry state he had signaled was in fact present when the flash occurred. If there was disagreement between these reports, the trial was discarded. The number of discarded trials proved to be very small.

Prior to formal data collection, each $S$ received at least 100 practice trials under rivalry conditions. During this time it was established that the suppression durations for both eyes for both Ss was of the order of $3 \mathrm{sec}$ or more. It was also established that $\mathrm{Ss}$ tended to depress the switch as soon as the designated nonsuppression state occurred.

During the formal data collection, 80 trials were run in a single session. Within the session, the order of occurrence of the suppression states (nonsuppression or suppression), the eye to be stimulated (left eye or right eye) and the position of the test flash (up or down) were randomized, with the restriction of equal occurrence. A total of five sessions was run in order to secure 100 trials under each combination of suppression and eye condition. The effect of all these randomizations was to make it impossible for $S$ to predict the eye and the rivalry state in which the test flash was going to occur on any given trial. The time between trials was approximately $10 \mathrm{sec}$. After each set of 20 trials was run, Ss were required to take a 5 -min rest. Before each session, Ss dark-adapted for $10 \mathrm{~min}$ and then aligned the rivalry targets for optimal fusion. This alignment was checked after every block of 20 trials.

\section{Results and Discussion}

The proportion of correct detections under all conditions was analyzed separately for each $S$ by analysis of variance. The main effect of suppression-nonsuppression was highly significant: For DC, $F(1 / 19)=24.36$, $\mathrm{p}<.001$; for JR, $\mathrm{F}(1 / 19)=36.28$, $\mathrm{p}<.001$. The analysis also revealed a significant interaction for the comparison of Right Eye/Left Eye by Suppression-Nonsuppression for JR: $F(1 / 19)=10.52, p<.01$. This comparison was not significant for DC.

Table 1 contains the proportion of correct detections for each $S$ in each eye under all conditions. Comparisons of the detection scores under suppression and nonsuppression conditions for each eye and for each $S$ were made by sign test for matched pairs. The significance level of these comparisons is indicated in the table.

Note that the proportion of nonsuppression detections for the left eye for both $S s$ is considerably greater than that of the left-eye monocular control condition detections, while for the right eye the nonsuppression detections are quite close to the right-eye monocular control condition detections. There is no obvious explanation for the increase of the left-eye nonsuppression detections. It is possible that this increase simply represents a less reliable estimation of the left-eye monocular threshold. Recall that the left-eye monocular thresholds were obtained by manipulating luminance increments rather than duration increments, and that the test flashes occurred upon a white rivalry target rather than upon a black one. The coritribution of these variables to the difference between the left- and right-eye monocular conditions is not clear, but at least it should be noted that the conditions for estimating monocular thresholds for the left and right eye were not strictly equivalent.

The main conclusion that these results 
Table 2

Experiment 2: Proportion of Correct Detections Under Suppression (SP) and Nonsuppressicn (NS) Conditions for Three Test-Flash Durations

\begin{tabular}{|c|c|c|c|c|c|}
\hline \multirow[b]{2}{*}{$\mathrm{S}$} & \multirow{2}{*}{$\begin{array}{c}\text { Test- } \\
\text { Flash } \\
\text { Duration } \\
\text { (Msec) }\end{array}$} & \multicolumn{2}{|c|}{$\begin{array}{l}\text { Left } \\
\text { Eye }\end{array}$} & \multicolumn{2}{|c|}{$\begin{array}{c}\text { Right } \\
\text { Eye }\end{array}$} \\
\hline & & SP & NS & SP & NS \\
\hline JR & $\begin{array}{l}55 \\
70 \\
85\end{array}$ & $\begin{array}{l}.66 \\
.66 \\
.62\end{array}$ & $\begin{array}{l}.88 \\
.96 \\
.92\end{array}$ & $\begin{array}{l}.46 \\
.48 \\
.52\end{array}$ & $\begin{array}{l}.62 \\
.62 \\
.78\end{array}$ \\
\hline$D C$ & $\begin{array}{l}50 \\
65 \\
80\end{array}$ & $\begin{array}{l}.52 \\
.58 \\
.68\end{array}$ & $\begin{array}{l}.80 \\
.80 \\
.90\end{array}$ & $\begin{array}{l}.52 \\
.48 \\
.58\end{array}$ & $\begin{array}{l}.62 \\
.78 \\
.86\end{array}$ \\
\hline KK & $\begin{array}{l}40 \\
55 \\
70\end{array}$ & $\begin{array}{l}.54 \\
.54 \\
.58\end{array}$ & $\begin{array}{l}.54 \\
.76 \\
.78 \\
\end{array}$ & $\begin{array}{l}.44 \\
.58 \\
.48\end{array}$ & $\begin{array}{l}.60 \\
.82 \\
.78\end{array}$ \\
\hline
\end{tabular}

support is that the suppression state significantly reduces the detectability of the test flash. Accordingly, this outcome is consistent with the hypothesis that suppression operates nonselectively upon all classes of test inputs.

\section{EXPERIMENT 2}

Experiment 2 was a direct extension of Experiment 1, with the incorporation of variable values of the test-flash energy in order to estimate the difference in thresholds between suppression and nonsuppression states. Specifically, thresholds were estimated by employing three levels of the test-flash energy within the context of the constant-stimulus method.

Subjects. Three Ss were employed: DC and IR, from Experiment 1, and a new $S$, KK, a 24-year-old female whose vision was corrected to normal by contact lenses. She had previous experience in observing rivalry, but was naive regarding the purpose of this experiment.

Apparatus. The apparatus was identical to that described in Experiment 1.

Design and procedure. The major difference between Experiments 1 and 2 was that, in the latter, three energy values of the test flash were employed. These values were manipulated by varying the duration of the test flash for both eyes for all three of the Ss. The baseline duration was the test-flash value that yielded approximately $75 \%$ correct detections for each eye of each $S$ under monocular conditions, as described in Experiment 1. Then, by increasing that monocular duration by $15 \mathrm{msec}$ a high value was established, one that would yield greater than $75 \%$ correct detections; conversely, by decreasing the baseline duration by $15 \mathrm{msec}$, a low value was established, one that would yield less than $75 \%$ correct detections. The decision to employ a 15-msec increment in duration for the high value and a 15 -msec decrement in duration for the low value of the test flash was based upon pilot tests that indicated that these values would produce detection levels useful in defining a psychometric function lying between chance performance and perfect performance.

The baseline values for JR and DC were the same combinations of duration and luminance values established for them in Experiment 1 . The baseline $75 \%$ value for KK was established for her right eye by manipulating the duration of the test flash and then for her left eye by holding that duration constant and manipulating the

\section{INCREMENT DETECTION THRESHOLDS FOR SUPPRESSION AND NONSUPPRESSION FOR THREE TEST-FLASH DURATIONS OVER EYES FOR THREE OBSERVERS}

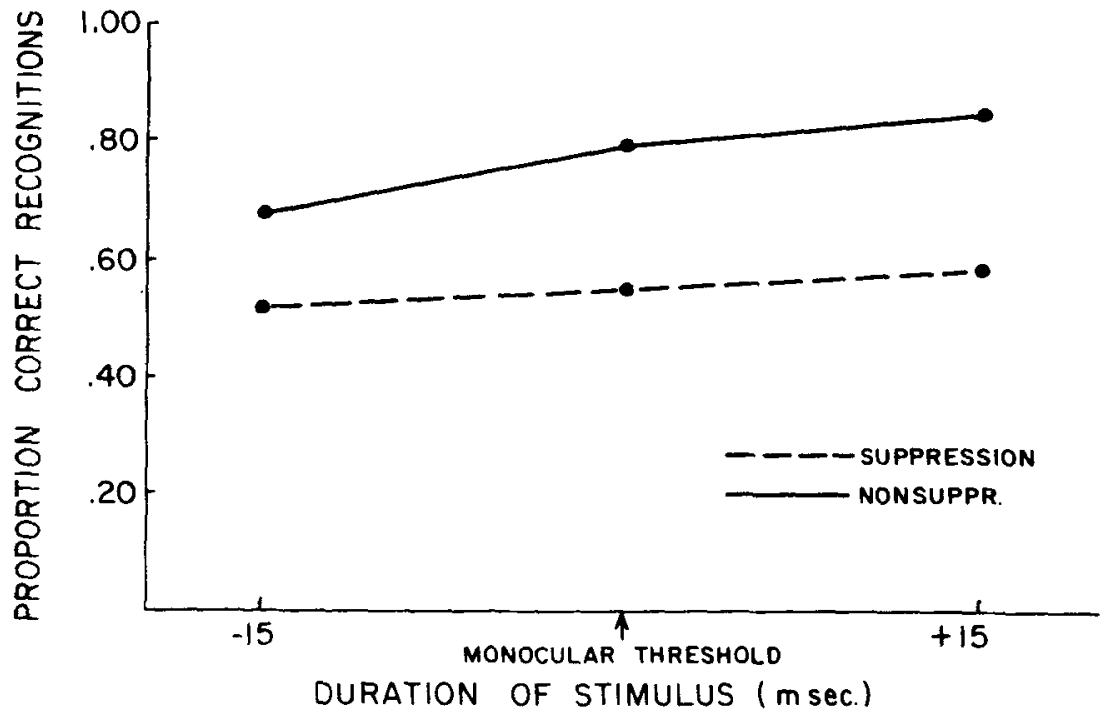

luminance level. These values were determined following the procedure described in Experiment 1. The baseline duration for $\mathrm{KK}$ was $55 \mathrm{msec}$; the luminance level for her right eye was $1.3 \mathrm{fL}$; the luminance level for her left eye was $2.2 \mathrm{fL}$. KK received 100 practice trials under conditions identical to those used in the actual experiment, and during this time it was established that the suppression durations for both her left eye and her right eye were in excess of $3 \mathrm{sec}$.

For each $S$, the three values of the test flash (high, baseline, and low) were administered following a randomized block design wherein the test-flash value defined the block. Within a block there were 20 trials consisting of equal numbers of suppression, nonsuppression, left- and right-eye presentations delivered in a predetermined random order. A total of 30 blocks, or 600 trials, was administered to each $\mathrm{S}$ so that 50 observations were obtained for each intersection of conditions, i.e., rivalry state $x$ eye $x$ test-flash value. Typically, four blocks were completed in a single session, using the same rest periods and checks on fusion alignment employed in Experiment 1.

\section{Results and Discussion}

In Table 2, the proportions of correct detections under suppression and nonsuppression conditions at each test stimulus value are given for each $\mathbf{S}$. In Fig. 2, the proportions of correct detections averaged over Ss and eyes have been plotted for the three stimulus values under suppression and nonsuppression conditions.

Analysis of variance of the data from each $S$ reveals a highly significant difference between suppression and nonsuppression conditions: for $\mathrm{KK}$, $F(1 / 9)=14.98, \quad p<.01 ;$ for JR, $F(1 / 9)=42.46, \quad p<.001 ;$ for $D C$, $\mathrm{F}(1 / 9)=32.91, \mathrm{p}<.001$.

Suppression and nonsuppression thresholds were estimated for each eye of each S separately. These estimates, obtained by a normal graphic solution described by Guilford (1954), are presented in Table 3. The median difference between suppression and nonsuppression duration thresholds, averaged over eyes and $\mathrm{Ss}$, equals $37 \mathrm{msec}$, a value corresponding to a $\log$ unit difference of .56 .

Fig. 2. The proportion of correct detections for both eyes of all Ss for the constant stimulus method used in Experiment 2. 
Table 3

Experiment 2: Estimated Thresholds (Medians and Standard Errors) Based on Normal Graphic Solution, in Msec of Test-Flash Duration

\begin{tabular}{|c|c|c|c|c|c|c|c|c|}
\hline \multirow[b]{3}{*}{$\mathbf{S}$} & \multicolumn{4}{|c|}{ Suppression } & \multicolumn{4}{|c|}{ Nonsuppression } \\
\hline & \multicolumn{2}{|c|}{ Left Eye } & \multicolumn{2}{|c|}{ Right Eye } & \multicolumn{2}{|c|}{ Left Eye } & \multicolumn{2}{|c|}{ Right Eye } \\
\hline & Mdn & SE & Mdn & SE & Mdn & SE & $\overline{M d n}$ & SE \\
\hline $\begin{array}{l}\mathrm{DC} \\
\mathrm{JR}\end{array}$ & 84 & 9.92 & 121 & 13.00 & 45 & 9.57 & 61 & 6.38 \\
\hline $\begin{array}{l}\text { JR } \\
\text { KK }\end{array}$ & $\begin{array}{r}85 \\
100\end{array}$ & $\begin{array}{r}9.57 \\
16.48\end{array}$ & $\begin{array}{c}100 \\
95.5\end{array}$ & $\begin{array}{r}10.63 \\
9.39\end{array}$ & $\begin{array}{l}53 \\
60\end{array}$ & $\begin{array}{l}3.37 \\
6.38\end{array}$ & $\begin{array}{l}68 \\
58\end{array}$ & $\begin{array}{l}9.75 \\
4.25\end{array}$ \\
\hline
\end{tabular}

It is worth noting that the nonsuppression detections obtained during data collection with the test flash duration established for the baseline $75 \%$ detection level do not depart significantly from the 75\% level for either eye for Ss DC and KK. For these two $\mathrm{Ss}$, the largest departure is a $7 \%$ increase (KK's right eye) and the smallest departure is a $1 \%$ increase (KK's left eye); the average difference is a $4 \%$ increase above the $75 \%$ baseline. In contrast, the corresponding nonsuppression detections for S JR depart considerably from the $75 \%$ baseline; there is a $13 \%$ decrease for his right eye and a 26\% increase for his left eye. Undoubtedly these variations reflect random variability owing in part to the small number of observations at each condition (i.e., $\mathrm{N}=50$ ). Yet, since an increase in detection was also observed for JR's left eye in Experiment 1, it is possible that such increases are a systematic aspect of his visual system. But in view of the results from $K K$ and $D C$, as well as from JR's right eye, it is not likely that the nonsuppression condition differs systematically from the monocular viewing under which the baseline $75 \%$ detection thresholds were established.

\section{GENERAL DISCUSSION}

The main conclusion supported by the data of both Experiments 1 and 2 is that detection of test flashes is significantly reduced during rivalry suppression. The major theoretical implication of this result is that it supports the hypothesis that suppression operates nonselectively upon many classes of test inputs.

The similarity between the baseline $75 \%$ detection thresholds obtained under monocular conditions and the corresponding thresholds obtained under nonsuppression phases of rivalry suggests that visual sensitivity is at a normal level during nonsuppression. Therefore, the elevation in threshold observed during suppression cannot be a relative one attributable to a supersensitive nonsuppression state, but rather must constitute a true reduction in sensitivity. This conclusion is in agreement with analogous threshold data for form recognition obtained by Fox and Check (1966).

The difference in threshold between suppression and nonsuppression of $.56 \mathrm{log}$ units is rather modest when compared to the changes in sensitivity observed in certain other visual phenomena, such as dark adaptation, and when compared with the powerful phenomenal effects of suppression, effects which can block from awareness for several seconds stimuli of great physical intensity. At present, little is known about the mechanism that produces suppression, but it is interesting to note that the threshold elevation of $.56 \mathrm{log}$ units produced by suppression is of the order of magnitude of threshold elevation obtained in visual masking (see Kahneman, 1968, for review). This may be fortuitous, or it may be a hint that similar processes underlie both masking and suppression.

\section{REFERENCES}

BLACKWELL, H. R. Psychophysical thresholds: Experimental studies of methods of measurement. Ann Arbor: Engineering Research Institute, University of Michigan, 1953.

CORNSWEET, T. N., \& PINSKER, H. M. Luminance discrimination of brief flashes under various conditions of adaptation. Journal of Physiology, 1965, 176, 294-310.

FOX, R., \& CHECK, R. Forced-choice form recognition during binocular rivalry. Psychonomic Science, 1966, 6, 471-472.

FOX, R., \& CHECK, R. Detection of motion during binocular rivalry suppression. Journal of Experimental Psychology, 1968, 78 , 388-395.

GERBRANDS, R., \& STEVENS, S. S. A high-intensity flash source. American Journal of Psychology, 1964, 77, 643-646.

GUILFORD, J. P. Psychometric methods New York: McGraw-Hill, 1954.

KAHNEMAN, D. Method, findings, and theory in studies of visual masking. Psychological Bulletin, 1968, 70, 404-425.

VERHOEFF, F. H. A new theory of binocular vision. Archives of Ophthalmology, 1935, 13, 151-175.

WOODWORTH, R. S. Experimental psychology. New York: Henry Holt, 1938.

\section{NOTES}

1. This report is based on the thesis submitted for the MA degree awarded to the senior author under the supervision of the junior author. The research was supported in part by a grant from the National Institutes of Health, NB 07619.

2. Address: Department of Psychology, Vanderbilt University, Nashville, Tennessee 37203.

(Accepted for publication November 14, 1969.) 